Check for updates

Cite this: RSC Adv., 2017, 7, 19764

Received 8th February 2017

Accepted 22nd March 2017

DOI: 10.1039/c7ra01603d

rsc.li/rsc-advances

\section{Super-stable non-woven fabric-based membrane as a high-efficiency oil/water separator in full $\mathrm{pH}$ range $\uparrow$}

\author{
Zhongwei Diao, ${ }^{a}$ Lei Wang, ${ }^{\text {*a }}$ Peng Yu, ${ }^{a}$ He Feng, ${ }^{a}$ Li Zhao, ${ }^{a}$ Wei Zhou ${ }^{a}$ \\ and Honggang Fu (D) *ab
}

\begin{abstract}
Oil separation is very important for avoiding environmental pollution originating from industrial wastewater and oil spillage, and therefore it is essential to explore various materials for oil separation. Herein, a polystyrene-coated non-woven fabric (PS/non-woven fabric) hybrid membrane with superoleophilicity has been synthesized by a facile, green and low-cost in situ polymerization reaction. The oil separation kinetic mechanism of the hybrid membrane was also studied. The present hybrid membrane possesses advantages of flexibility and high efficiency, and demonstrates great potential applications in the various environments and even under different harsh conditions, which makes it very promising for the treatment of oil pollution in practical applications.
\end{abstract}

\section{Introduction}

Oil pollution always causes serious environmental problems due to frequent oil spillage accidents and unsafe oily industry wastewater; therefore oil/water separation has become a worldwide task in recent years. ${ }^{1-4}$ Traditional oil/water separation methods, including use of oil skimmers, centrifugation, distillation and electrophoresis, are generally time-consuming, highcost and low-efficiency methods..$^{5,6}$ Therefore, researchers have focused on the development of novel technologies, which are easy-to-operate, highly-efficient and inexpensive, to assist in clean-up of oil/water mixtures. ${ }^{7-10}$

Because oil/water separation is essentially an interfacial issue, the exploration of novel strategies based on special wettability is deemed to be facile and effective. ${ }^{11}$ Since the superwettable coating mesh has been used to separate an oil/ water mixture, it has become a popular topic in the exploration of various materials based on special wettability oil/water separation in recent years. ${ }^{12-20}$ Membrane materials composed of inorganic composites, organic polymers or some organic/ inorganic hybrids, have been widely used as an effective separator for separating oil from sewage. ${ }^{15,17,19,20}$ It has been demonstrated that materials able to process both the superhydrophobicity of a water contact angle greater than $150^{\circ}$ and the superoleophilicity of an oil contact angle less than $10^{\circ}$ are

${ }^{a}$ Key Laboratory of Functional Inorganic Material Chemistry, Ministry of Education of the People's Republic of China, Heilongjiang University, Harbin 150080, China. E-mail:wanglei0525@126.com; fuhg@vip.sina.com

${ }^{b}$ National Center for International Research on Catalytic Technology, China

$\dagger$ Electronic supplementary information (ESI) available: Photographs and images. See DOI: $10.1039 / \mathrm{c} 7 \mathrm{ra} 01603 \mathrm{~d}$ vital for efficient oil separation from oil/water mixtures. ${ }^{21}$ The separation principle is that the oil phase spreads and penetrates through the membrane easily, while simultaneously, the water phase is hindered. ${ }^{22-27}$ Most of these membrane materials are synthesized by modifying different materials on various porous metal substrates, such as copper grid, nickel grid and stainless steel. ${ }^{\mathbf{8 , 1 4 , 2 2 , 2 4}}$ As a result, the abovementioned membranes are generally applied in a neutral environment for oil/water separation, and it is difficult to realize the separation operation in another harsh environment, such as strong acid or strong alkali oily wastewater. ${ }^{28-30}$ In addition, the high-cost, low-efficiency and hard characteristics of these substrates would limit their further practical applications. ${ }^{31-38}$ Therefore, it is urgent to develop novel stable membrane materials possessing attractive performance and a reasonable price, which can be used in harsh environments, particularly in strong acid and strong alkali. ${ }^{20}$

Non-woven fabrics directly use polymer slice, short fibers or filament fibers woven together into net structures through air flow or machines, followed by the water thorn, acupuncture or hot rolling reinforcement to finish the fabrication. Owing to the flexible, low-density, high-porosity, hydrophobicity and lowcost, non-woven fabrics are expected to be used as a promising substance for the preparation of high efficiency membrane materials..$^{39,40}$ In addition, polymer modification could promote the oil separation efficiency by enhancing superhydrophobic properties, and hence it may be the best option to modify non-woven fabrics with polymers for fabricating high-efficiency membranes. ${ }^{41-46}$

Herein, we have developed a facile and scalable strategy for the synthesis of polystyrene (PS)-coated non-woven fabric hybrid membrane material with superoleophilicity via an in situ 
polymerization reaction, which could be explored as a useful filter separator for oil from an oil/water mixture in harsh environments. The synthetic hybrid membrane could selectively adsorb and separate not only the light oils ( $n$-heptane, acetone, toluene, ether and octane) but also the heavy oils (chloroform and dichloromethane), with a high absorption capacity of about 12-26 times the weight of the hybrid membrane. Furthermore, it can show a high separation efficiency of above $97 \%$ at the $\mathrm{pH}$ values of 1-14, and a stable recyclability even after 10 cycles, maintaining its initial performance. The oil separation kinetic mechanism follows the pseudo-first-order kinetics equation.

\section{Experimental}

\subsection{Synthesis of hybrid membranes}

The initial nonwoven fabric composed of polyester (polyester) with a thickness of $0.5 \mathrm{~mm}$ was purchased from Shandong Hengruitong New Material Engineering Limited Company. Styrene $\left(\mathrm{C}_{8} \mathrm{H}_{6}\right)$ with analytic purity was purchased from Tianjin Zhiyuan Chemical Reagent Limited Company. The polystyrene (PS)/nonwoven fabric hybrid membrane was synthesized by a facile polymerization reaction. Typically, a piece of non-woven fabric with a size of $10 \mathrm{~cm} \times 10 \mathrm{~cm}$ was soaked in distilled water for $2 \mathrm{~h}$ and then soaked in ethanol solution for another $2 \mathrm{~h}$. Subsequently, the treated non-woven fabric was dried at $80^{\circ} \mathrm{C}$. In the polymerization reaction, the treated non-woven fabric was put into a three-necked bottle containing $120 \mathrm{~mL}$ styrene solution, and refluxed for $6 \mathrm{~h}$ at $70{ }^{\circ} \mathrm{C}$ to finish the polymerization process of styrene on the surface of non-woven fabrics. Finally, polystyrene (PS)/non-woven fabric hybrid membrane was obtained.

\subsection{Characterizations}

Scanning electron microscopy (SEM) micrographs were tested by a Hitachi S-4800 instrument operating at $5 \mathrm{kV}$. FT-IR spectra were recorded on a NICOLETiS10 using $\mathrm{KBr}$ pellets. The contact angles (CA) were examined using an OCA20 contact angle tester.

\subsection{Selective absorption and separation of oil}

Oil/water mixtures were prepared by mixing water and oil in a volume ratio of $1: 2 \mathrm{v} / \mathrm{v}$ for heavy oils and $2: 1$ for light oils. To clearly observe the absorption and separation processes of oils, the chloroform, acetone, toluene, ether, octane and dichloromethane are dyed by Rhodamine $\mathrm{B}$, and $n$-heptane is colored with Sudan Red. For oil absorption, the PS/non-woven fabric hybrid membranes were immersed in $45 \mathrm{~mL}$ oil/water mixture. The capacity to absorb oil was tested by the immersion of the membrane in the oil/water mixture until saturation. For oil/ water separation, the PS/non-woven fabric hybrid was used as the membrane, and $20 \mathrm{~mL}$ oil/water mixture was poured slowly into a test tube to pass through the membrane. Then, the separated oil and water were collected into separate beakers.

\section{Results and discussion}

FT-IR spectra could provide detailed information about organic groups. The synthetic PS/non-woven fabric hybrid membrane

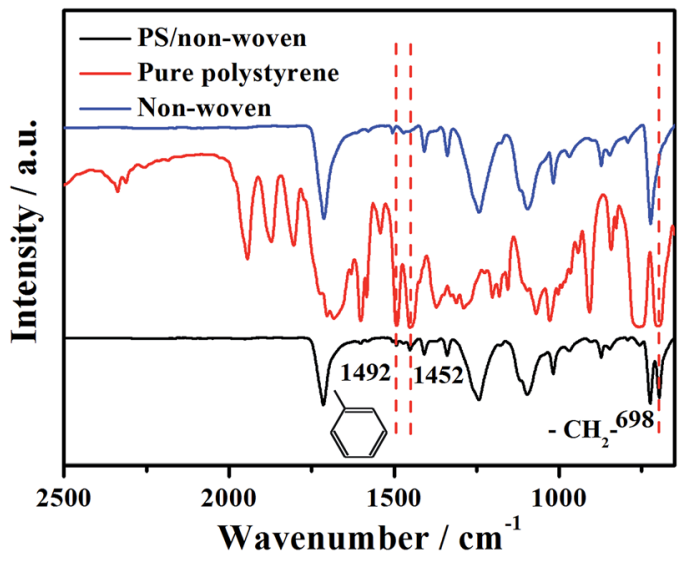

Fig. 1 FT-IR spectra for blank non-woven fabrics, pure polystyrene and PS/non-woven fabric hybrid membrane.

was first characterized by FT-IR spectroscopy, and the corresponding studies of non-woven fabrics and pure polystyrene were also carried out for comparison. The blank non-woven fabric has no evident characteristic peak (Fig. 1). Comparing IR spectra of PS/non-woven with pure polystyrene, it can be seen that the PS/non-woven fabric hybrid membrane exhibits two characteristic peaks of polystyrene at 1492, 1452 and $698 \mathrm{~cm}^{-1}$, respectively, which are ascribed to the $-\mathrm{CH}_{2}$ - bending vibration and the phenyl vibration absorption. ${ }^{32}$ This illustrates the successful modification of PS on the non-woven fabric substrate.

Scanning electron microscopy (SEM) is a common technology for the analysis of the micromorphology of materials. As shown in Fig. 2A and B, the blank non-woven fabric is composed of uniform and smooth surface long fibers with a diameter of about $12 \mu \mathrm{m}$. After modifying by PS, it can be seen that the surfaces of these long fibers are relatively rough (Fig. 2C and D), indicating the PS could adhere on the surface of the non-woven fabric layer to form oil-wet hydrophobic material.
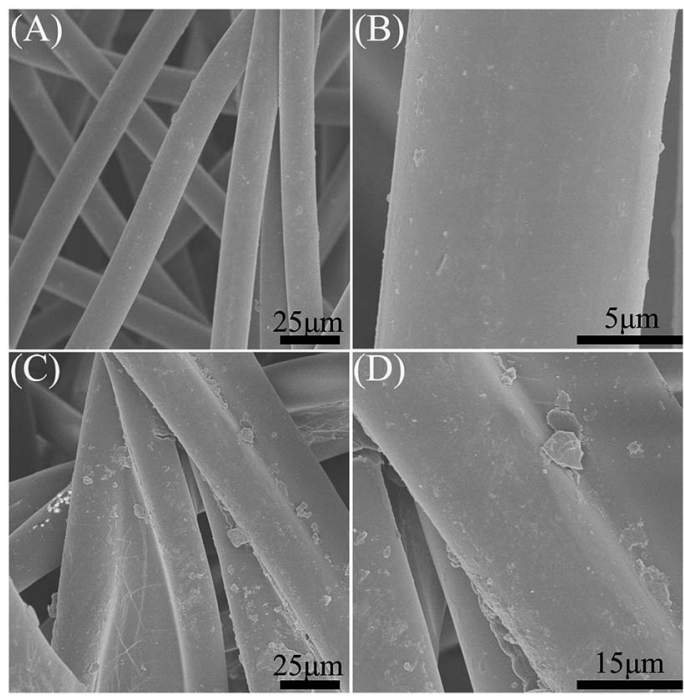

Fig. 2 SEM images of (A, B) blank non-woven fabric and (C, D) PS/ non-woven fabric hybrid membrane. 
(A)

(B)

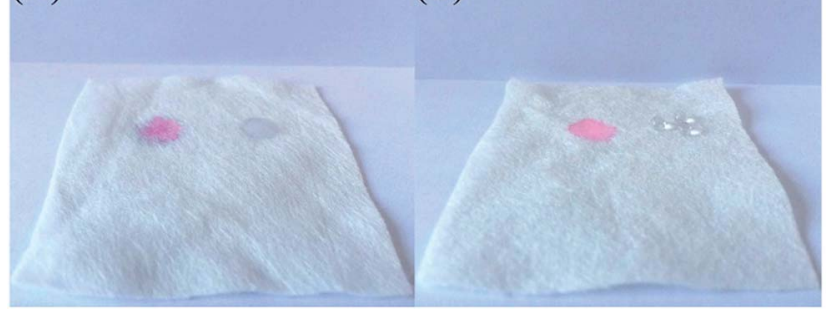

Fig. 3 Optical images of an oil droplet (left, $20 \mu \mathrm{L}$ chloroform colored with Rhodamine B) and water droplet (right, $20 \mu \mathrm{L}$ deionized water) on a non-woven fabric before (A) and after (B) modification by PS

The PS content loading on non-woven fabric is about $3.5 \mathrm{mg}$ $\mathrm{cm}^{-2}$, calculated based on a weighting method. In the macroscopic view, as shown in Fig. 3, there is no significant difference between non-woven fabrics before and after modifying by PS.

To investigate the surface property change of non-woven fabrics before and after modifying by PS, the special wettability of the synthetic materials was also studied. In these cases, an oil droplet of $20 \mu \mathrm{L}$ chloroform colored with Rhodamine B and a water droplet of $20 \mu \mathrm{L}$ deionized water were dropped on the surface of non-woven fabric before and after modifying by PS. As can be seen from Fig. 3A, the blank non-woven fabric exhibited superamphiphilic properties for oil and water. On the contrary, after modifying by PS, the synthetic hybrid membrane exhibited superhydrophobicity, as illustrated in Fig. 3B. The hydrophobic or hydrophilic wettability of a material surface is mainly dependent on its surface exposed groups, which is determined by the surface chemical composition; therefore, the PS on the material surface plays a vital role for the superhydrophobic properties of the hybrid membrane.

Contact angle (CA) measurement is an effective method for characterizing the hydrophobic or hydrophilic wettability of membrane materials. The water contact angle (WCA) for PS/ non-woven fabric hybrid membrane is about $173.6^{\circ}$, as shown in Fig. 4A, implying the superhydrophobicity of the synthetic hybrid membrane. Moreover, the corresponding CA for chloroform is about $0^{\circ}$ (Fig. S1 $\dagger$ ). It could further indicate good superoleophilicity of the synthetic PS/non-woven fabric hybrid

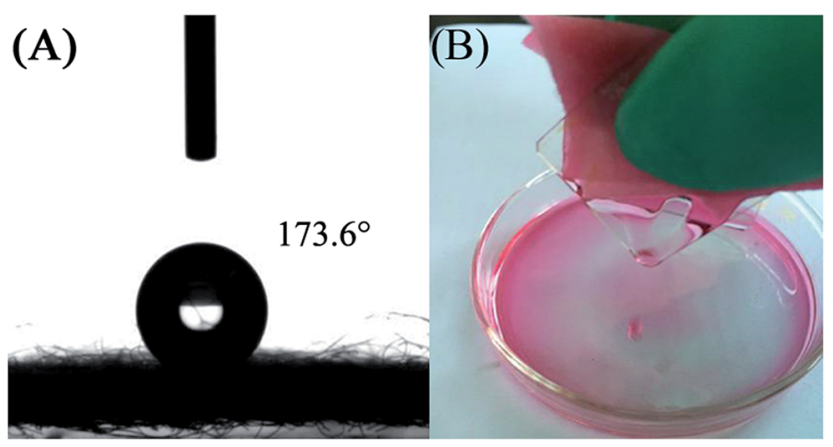

Fig. 4 (A) Photograph of a water droplet on PS/non-woven fabric hybrid membrane with a CA of $173.6^{\circ}$. (B) Optical image of chloroform (colored with Rhodamine B) adsorption for PS/non-woven fabric hybrid membrane. membrane, which can be used as a high efficiency oil separator from an oil/water mixture. In the case of oil absorption, the chloroform is colored with Rhodamine B for clear observation of the oil separation phenomenon. Fig. 4B illustrates that the absorption of chloroform could easily be observed via extrusion of the hybrid membrane owing to the flexible characteristic of non-woven fabric, which demonstrates the recyclability of these types of materials. The CA of the blank non-woven fabric with water and chloroform for 3 different experiments was also tested, and the corresponding videos have been provided (Video 1,2 and $3 \dagger$ are the WCA for the first, second and third time, respectively, Video 4, 5 and $6 \dagger$ are the chloroform CA for the first, second and third time, respectively). It can be seen that the blank non-woven fabric exhibits superamphiphilic properties for oil and water, further indicating the importance of PS modification for the change of surface properties.

The universality for this type of membrane materials to adsorb various oils from oil/water mixtures is very important in practical application because there are various types of oils that exist simultaneously in wastewater. Based on the above consideration, further experiments using various types of oils including chloroform, $n$-heptane, acetone, toluene, ether, octane and di-chloromethane have been carried out (Fig. 5A). It was confirmed that the synthetic hybrid membrane exhibited an excellent superoleophilic performance (the absorption capacity is about 12-26 times the weight of the hybrid membrane) towards various types of oils mentioned above. Clearly, the hybrid membrane displays the best absorption performance towards chloroform. Fig. 5C-E illustrates the sorption processes of chloroform (colored with Rhodamine B). A droplet of dyed chloroform was put into the bottom of an aqueous solution (water is colored by methylene blue), and then a piece of PS/non-woven fabric hybrid membrane was brought into contact with the chloroform droplet. The chloroform

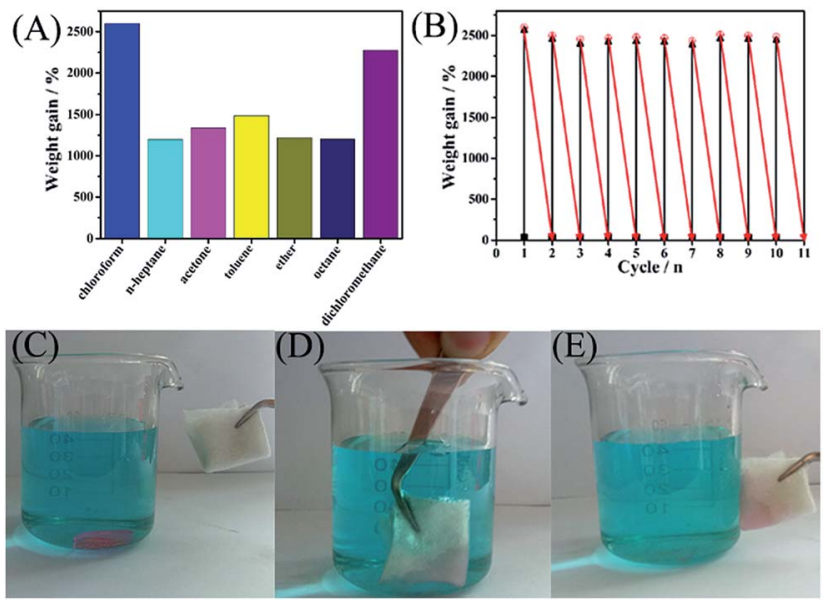

Fig. 5 (A) The adsorption capacity of PS/non-woven fabric hybrid membrane for different oils from oil/water mixtures. (B) The 10 cycles adsorption performances of PS/non-woven fabric hybrid membrane for chloroform. (C-E) The illustration of chloroform (colored by Rhodamine B) adsorption processes in aqueous solution (water is colored by methylene blue) for PS/non-woven fabric hybrid membrane. 
droplet was completely adsorbed in mere seconds, indicating good absorption for chloroform. Notably, the hybrid membrane could be reused after a simple and convenient squeezing. As shown in Fig. 5B, the PS/non-woven fabric hybrid membrane could retain an absorption capacity of above $97.0 \%$ of its initial absorption capacity after 10 cycles, which demonstrates the favorable recyclability of the hybrid membrane materials. It should be noted that the coated PS could not be dissolved by chloroform, acetone, or toluene during the oil/water separation or after the adsorption test, and hence a stable performance could be obtained even after 10 cycles.

The superhydrophobic property of the synthetic hybrid membrane is feasible and desirable for oil/water separation, and hence the corresponding experiments have been studied. For oils, chloroform and dichloromethane, with higher densities than that of water, the used pictorial views of the oil/water separation system are displayed in Fig. 6A. The oils placed themselves in the lower region of the solution due to gravity, and therefore chloroform could move rapidly through the hybrid membrane and water was intercepted due to the superhydrophobic properties of the synthetic materials. The chloroform and dichloromethane separation rates are about 3500 and $3400 \mathrm{~L} \mathrm{~m}^{-2} \mathrm{~h}^{-1}$, respectively, which is better than that of the reported polymer coated PVDF-based membrane materials. $^{22,23,32}$ The volume change of the chloroform before and after separation from the oil/water mixture is measured using a graduated cylinder (Fig. S2 $\dagger$ ). The excellent separation performance should be attributed to the smaller oil CA of the synthetic hybrid membrane. Owing to the fact that the modified nonwoven fabric can adsorb some Rhodamine B, a lighter color could be observed after adsorption.

As is known, the polymerization degree and loading content of polymer on the substrate of non-woven fabric affect the separation performance of the synthetic hybrid membrane seriously, and therefore a series of experiments on polymerization time of PS have been investigated. As shown in Fig. 7, the loading content of PS polymer on non-woven fabric is increased gradually along with the increase in polymerization time (loading content is about $1.9-3.8 \mathrm{mg} \mathrm{cm}^{-2}$ for polymerization time in the range of 4-7 h). The WCA is clearly increases when the polymerization time is increased. The water contact angles

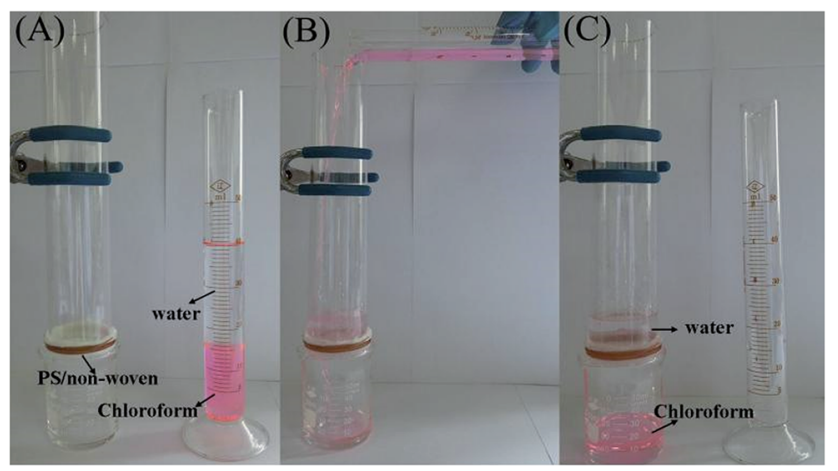

Fig. 6 Optical images of the heavy oil separation from an oil/water mixture (colorless for water and pink for chloroform dyed by Rhodamine B). for $4 \mathrm{~h}$ and $5 \mathrm{~h}$ are about $131.7^{\circ}$ and $136.5^{\circ}$, respectively. Nevertheless, when the polymerization time is increased to $6 \mathrm{~h}$ and $7 \mathrm{~h}$, the corresponding WCA is about $173.6^{\circ}$ and $173.8^{\circ}$, respectively. It is recognized that superhydrophobic materials have a water contact angles of more than $150^{\circ}$. That is to say, the increase in polymerization time brings a qualitative change for the synthetic hybrid membrane from hydrophilicity to superhydrophobicity. It should be noted that the hybrid membrane has no oil separation property from an oil/water mixture when the polymerization time is less than $6 \mathrm{~h}$, which is attributed to a small amount of PS on the substrate that cannot completely cover the surface of the non-woven fabric, and the hybrid membrane, thus, exhibits the hydrophilic and lipophilic characteristics of non-woven fabric. The separation rate for the $7 \mathrm{~h}$ polymerization time of the hybrid membrane is much lower than that of the $6 \mathrm{~h}$ polymerization time $\left(2500 \mathrm{~L} \mathrm{~m}^{-2} \mathrm{~h}^{-1} v s\right.$. $3500 \mathrm{~L} \mathrm{~m}^{-2} \mathrm{~h}^{-1}$ for chloroform). This phenomenon should be ascribed to the long polymerization time that results in a higher loading content of PS on the substrate, leading to a pore barrage of the non-woven fabric resulting in a slow separation rate. The abovementioned analyses demonstrate that the $6 \mathrm{~h}$ polymerization time is the optimal synthetic condition in our study.

The separation performance of this hybrid membrane in various harsh environments is of the highest importance in practical applications. The separation of chloroform from a chloroform/water mixture with a $\mathrm{pH}$ values ranging between 1 and 14 using PS/non-woven fabric hybrid membrane was studied. As shown in Fig. 8, all the water contact angles for the synthetic hybrid membrane were above $170^{\circ}$ for various $\mathrm{pH}$ values, indicating that the superhydrophobicity could be maintained in a harsh environment. Simultaneously, the synthetic PS/non-woven fabric hybrid membrane maintained a consistent performance for chloroform separation from a chloroform/water mixture under the conditions of $\mathrm{pH}=1-14$. The results revealed that our present hybrid membranes could be used in various complex environments, particularly for strong acid and alkali, and could satisfy the requirements for treating the oily wastewater originating from different industries and oceans.

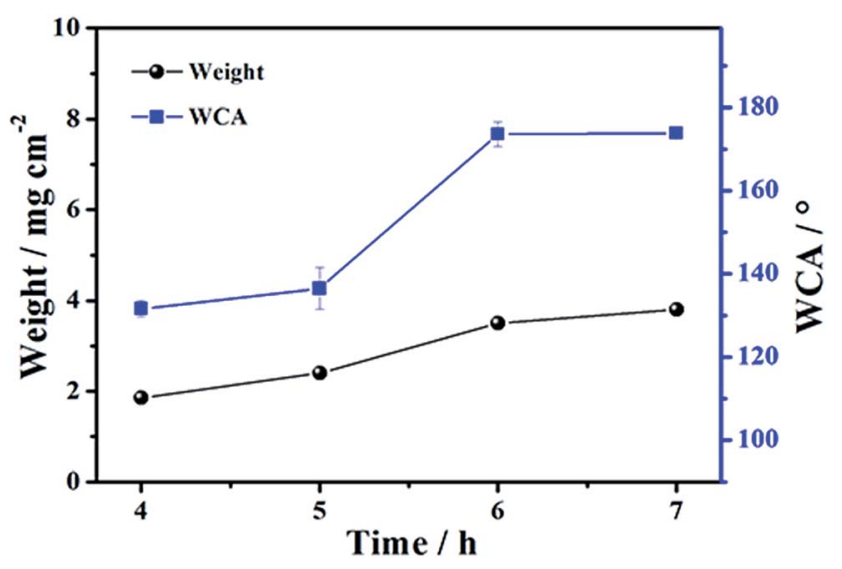

Fig. 7 The changes of loading content (left) and WCA (right) along with the polymerization time of PS. The corresponding error bar of the contact angle is displayed. 


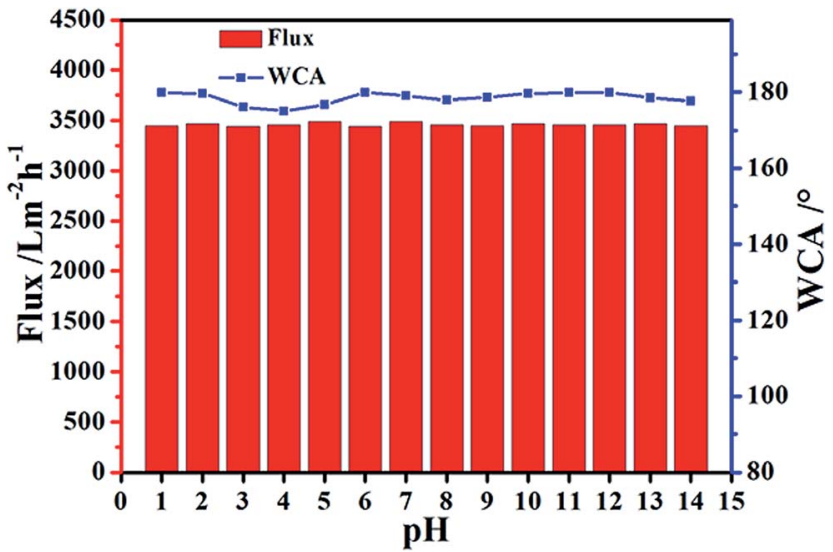

Fig. 8 The changes of chloroform separation performance (left) and WCA (right) of PS/non-woven fabric hybrid membranes from chloroform/water mixtures under different $\mathrm{pH}$ values. The error bars for all the WCAs are also shown.

The study of separation kinetic mechanisms is significant for analyzing the separation process of different materials. For this purpose, we used the chloroform separation as the case to investigate the kinetic mechanism of oil for the synthetic hybrid membrane. The pseudo-first-order rate equation is as follows: ${ }^{47}$

$$
\log \left(V_{\mathrm{e}}-V_{t}\right)=\log V_{\mathrm{e}}-\frac{k_{1}}{2.303} t
$$

where $V_{\mathrm{e}}$ and $V_{t}$ are the separated amounts $\left(\mathrm{L} \mathrm{min}^{-1}\right.$ ) of chloroform at equilibrium and at any time $t$, respectively, and $k_{1}$ is the rate constant of pseudo-first-order separation $\left(\mathrm{min}^{-1}\right)$. The $V_{\mathrm{e}}$ and rate constants $k_{1}$ were calculated from the slope and intercept of the linear of $\log \left(V_{\mathrm{e}}-V_{t}\right)$ vs. $t$ (see Fig. 9), respectively. Notably, the calculated equilibrium separation amount value of $V_{\mathrm{e}}$ (cal.) must be in agreement with the experimental $V_{\mathrm{e}}$ (exp.) values. ${ }^{34}$ For the separation of chloroform from the chloroform/water mixture, the correlation coefficient value $\left(R^{2}\right)$ is as high as 0.9983 , while simultaneously, the experimental $V_{\mathrm{e}}$

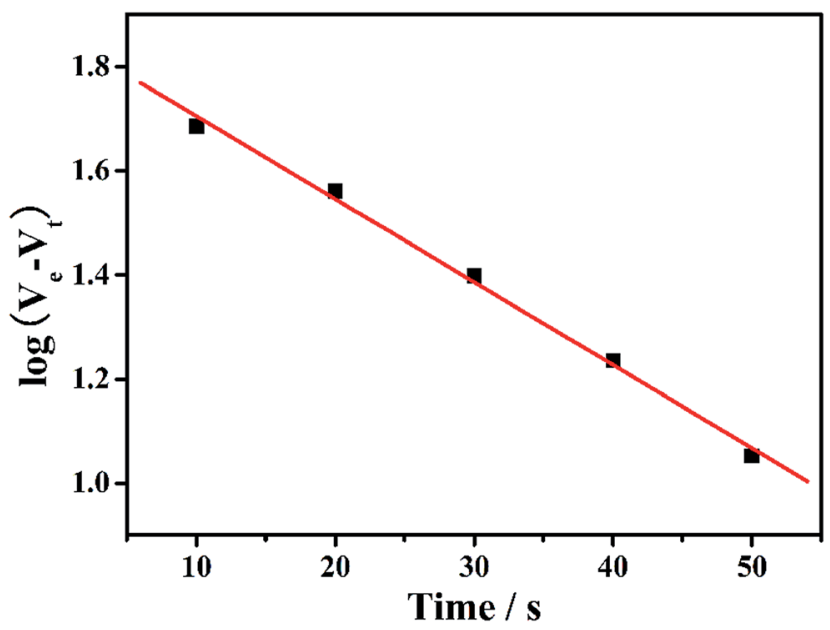

Fig. 9 The pseudo-first-order linear plots of chloroform separation from oil/water mixtures in different times on PS/non-woven fabric hybrid membrane. value is close to the calculated value obtained from the linear plots (61.6 vs. 58.1). It is suggested that the separation process of chloroform follows pseudo-first-order kinetics.

To perform different types of oil separations from oily wastewater, the separation properties of the separation materials for low density oil are as important as those of the high density oil. The corresponding studies for separations of $n$ heptane, acetone, toluene, ether and octane were also investigated. Owing to all these types of oils exhibiting a lower density than water, the dumping and filtering experimental equipment, as shown in Fig. 10A-C, was used here. The separation of $n$ heptane (dyed by Sudan Red) from the $n$-heptane/water mixture in Fig. 10A-C indicated that $n$-heptane could penetrate through the hybrid membrane, whereas the water could steadily stay on the surface of the hybrid membrane. The entire separation process could be finished within a short time, and the corresponding oil/water separation efficiency was calculated using the following equation: ${ }^{26,29}$

$$
E_{\mathrm{ff}}=\left(1-C_{\mathrm{p}} / C_{\mathrm{o}}\right) \times 100 \%
$$

where $C_{\mathrm{o}}$ and $C_{\mathrm{p}}$ are calculated based on the oil volume in the initial oil/water mixture and the filtered mixture, respectively, using a small measuring cylinder. As shown in Fig. 10D, the synthetic hybrid membranes exhibit excellent performance with more than $97 \%$ separation efficiency for $n$-heptane by measuring the volume change (Fig. S3†). For other oils, such as acetone, toluene, ether and octane, the corresponding separation efficiencies are about $98 \%, 97 \%, 97 \%$ and $96 \%$, respectively. It is demonstrated that the synthetic hybrid membrane

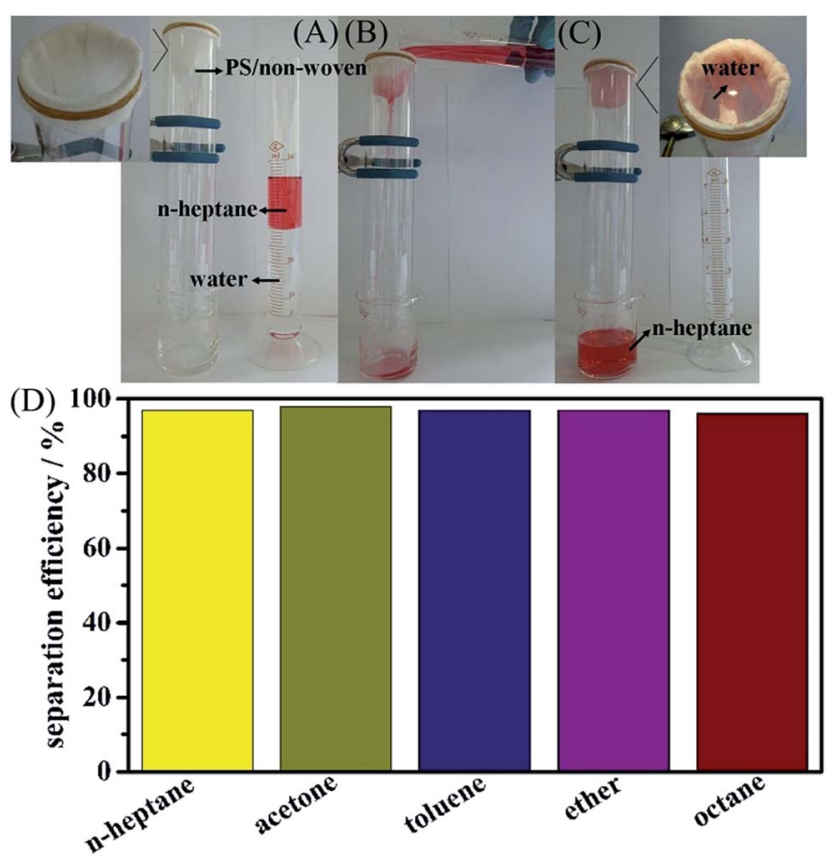

Fig. 10 (A-C) Optical images of the light oil $n$-heptane separation from $n$-heptane/water mixture ( $n$-heptane colored with Sudan Red). (D) The separation efficiency of $n$-heptane, acetone, toluene, ether and octane from oil/water mixtures by using PS/non-woven fabric hybrid membranes. 
could be used not only for the separation of high density oils but also for the separation of low density oils.

\section{Conclusions}

In summary, we have developed a facile and feasible approach to synthesize a superoleophilic PS/non-woven fabric hybrid membrane for highly efficient oil/water separation. The synthetic hybrid membrane could be used as a separator for oils from oil/water mixtures, including light oils and heavy oils. The membrane can be easily recycled by extrusion, owing to the flexible nature of non-woven fabrics, and the oil absorption performance could still be retained as their initial capacity after 10 cycles. The oil separation efficiency of the synthetic hybrid membranes for various oils is above $97 \%$, and the corresponding separation kinetic mechanism for oil follows the pseudo-first-order kinetics equation. More importantly, the hybrid membrane can be applied in various complex environments, particularly for strong acid and alkali, demonstrating the potential applications in practical situations. Our present strategy provides a simple way to fabricate the hybrid membrane materials for oil separation from oil/water mixtures with low cost and high efficiency.

\section{Acknowledgements}

We gratefully acknowledge the support of this research by the National Natural Science Foundation of China (21631004, 21371053, 21401048, 51672073), the Project for Foshan Innovation Group (2014IT100062), the application technology research and development projects in Harbin (2013AE4BW051), the University Nursing Program for Young Scholars with Creative Talents in Heilongjiang Province (UNPYSCT-2016016), the Harbin science and technology innovation talents research Foundation (2015RAQXJ057).

\section{Notes and references}

1 B. Cortese, D. Caschera, F. Federici, G. M. Ingo and G. Gigli, J. Mater. Chem. A, 2014, 2, 6781.

2 P. Kothary, N. Yanumet and E. A. O'Rear, Fibers Polym., 2013, 5, 710.

3 K. Lee, J. Hwang and Y. Ahn, Bull. Korean Chem. Soc., 2014, 5, 1545.

4 F. Lu, Y. N. Chen, N. Liu, Y. Z. Cao, L. X. Xu, Y. Wei and L. Feng, RSC Adv., 2014, 4, 32544.

5 L. Wu, J. P. Zhang, B. C. Li and A. Q. Wang, Polym. Chem., 2014, 5, 2382.

6 J. B. Fan, Y. Y. Song, S. T. Wang, J. X. Meng, G. Yang, X. L. Guo, L. Feng and L. Jiang, Adv. Funct. Mater., 2015, 25, 5368.

7 D. T. Ge, L. L. Yang, C. B. Wang, E. Lee, Y. Q. Zhang and S. Yang, Chem. Commun., 2015, 51, 6149.

8 A. Raza, B. Ding, G. Zainab, M. El-Newehy, S. S. Aldeyab and J. Yu, J. Mater. Chem. A, 2014, 2, 10137.
9 Y. lu, S. Sathasivam, J. L. Song, F. Chen, W. J. Xu, C. J. Carmalt and I. P. Parkin, J. Mater. Chem. A, 2014, 2, 11628.

10 L. Y. Liu, C. Chen, S. Y. Yang, H. Xie, M. G. Gong and X. L. Xu, Phys. Chem. Chem. Phys., 2016, 18, 1317.

11 G. Wang, Y. He, H. Wang, L. Zhang, Q. Y. Yu, S. S. Peng, X. D. Wu, T. H. Ren, Z. X. Zeng and Q. J. Xue, Green Chem., 2015, 17, 3093.

12 J. Li, D. M. Li, Y. X. Yang, J. P. Li, F. Zha and Z. Q. Lei, Green Chem., 2016, 18, 541.

13 J. Li, L. Yan, H. Y. Li, W. J. Li, F. Zha and Z. Q. Lei, J. Mater. Chem. A, 2015, 3, 14696.

14 S. Yu and Z. G. Gu, RSC Adv., 2015, 5, 107880.

15 Z. L. Chu, Y. J. Feng and S. Seeger, Angew. Chem., Int. Ed., 2015, 54, 2328.

16 C. R. Crick, J. A. Gibbins and I. P. Parkin, J. Mater. Chem. A, 2013, 1, 5943.

17 M. M. Tao, L. X. Xue, F. Liu and L. Jiang, Adv. Mater., 2014, 26, 2943.

18 J. T. Wang and Y. H. Chen, J. Appl. Polym. Sci., 2015, 10, 42614.

19 M. N. Kavalenka, A. Hopf, M. Schneider, M. Worgull and H. Holscher, RSC Adv., 2014, 4, 31079.

20 J. Li, L. Yan, H. Y. Li, J. P. Li, F. Zha and Z. Q. Lei, RSC Adv., 2015, 5, 53802.

21 B. Y. Chen, J. H. Qiu, E. Sakai, N. Kanazawa, R. Liang and H. X. Feng, ACS Appl. Mater. Interfaces, 2016, 8, 17659.

22 Z. G. Xu, Y. Zhao, H. X. Wang, X. G. Wang and T. Lin, Angew. Chem., Int. Ed., 2015, 54, 4527.

23 W. B. Zhang, Y. Z. Zhu, X. Liu, D. Wang, J. Y. Li, L. Jiang and J. Jin, Angew. Chem., Int. Ed., 2014, 53, 856.

24 Z. Y. Luo, K. X. Chen, Y. Q. Wang, J. H. Wang, D. C. Mo and S. S. Lyu, J. Phys. Chem. C, 2016, 120, 12685.

25 Q. L. Ma, H. F. Cheng, A. G. Fane, R. Wang and H. Zhang, Small, 2016, 16, 2186.

26 N. W. Gao, Y. Q. Fan, X. J. Quan, Y. W. Cai and D. W. Zhou, J. Mater. Sci., 2016, 51, 6379.

27 R. Du, Q. L. Feng, H. Y. Ren, Q. C. Zhao, X. Gao and J. Zhang, J. Mater. Chem. A, 2016, 4, 938.

28 V. A. Ganesh, A. S. Ranganath, A. Baji, H. Wong, H. Raut, R. Sahay and S. Ramakrishn, Macromol. Mater. Eng., 2016, 301, 812 .

29 C. M. Xiao, L. X. Si, Y. M. Liu, G. Q. Guan, D. H. Wu, Z. D. Wang and X. G. Hao, J. Mater. Chem. A, 2016, 4, 8080.

30 J. T. Wang, F. L. Han and S. C. Zhang, Sep. Purif. Technol., 2016, 164, 138.

31 X. D. Lin, M. Yang, H. Jeong, M. Chang and J. Hong, J. Membr. Sci., 2016, 506, 22.

32 W. B. Zhang, Z. Shi, F. Zhang, X. Liu, J. Jin and L. Jiang, Adv. Mater., 2013, 25, 2071.

33 Q. H. Zeng, Q. L. Liu, I. Broadwell, A. M. Zhu, Y. Xiong and X. P. Tu, J. Membr. Sci., 2010, 349, 237.

34 J. Febrianto, A. N. Kosasih, J. Sunarso, Y. Ju, N. Indraswati and S. Ismadji, J. Hazard. Mater., 2009, 162, 616.

35 L. R. Shi, K. Chen, R. Du, A. Bachmatiuk, M. H. Rümmeli, K. W. Xie, Y. Huang, Y. Y. Zhang and Z. F. Liu, J. Am. Chem. Soc., 2016, 138, 6360. 
36 B. Wang, J. Li, G. Y. Wang, W. X. Liang, Y. B. Zhang, L. Shi, Z. G. Guo and W. M. Liu, ACS Appl. Mater. Interfaces, 2013, 5, 1827.

37 Y. Z. Cao, X. Y. Zhang, L. Tao, K. Li, Z. X. Xue, L. Feng and Y. Wei, ACS Appl. Mater. Interfaces, 2013, 5, 4438.

38 Y. W. Shang, Y. Si, A. Raza, L. P. Yang, X. Mao, B. Ding and J. Y. Yu, Nanoscale, 2012, 4, 7847.

39 Q. Wen, J. C. Di, L. Jiang, J. H. Yu and R. R. Xu, Chem. Sci., 2013, 4, 591.

40 X. F. Gao, L. P. Xu, Z. X. Xue, L. Feng, J. T. Peng, Y. Q. Wen, S. T. Wang and X. J. Zhang, Adv. Mater., 2014, 26, 1771.
41 J. C. Gu, P. Xiao, J. Chen, F. Liu, Y. J. Huang, G. Y. Li, J. W. Zhang and T. Chen, J. Mater. Chem. A, 2014, 2, 15268. 42 N. Chen and Q. M. Pan, ACS Nano, 2013, 7, 6875.

43 P. C. Chen and Z. K. Xu, Sci. Rep., 2013, 3, 2776.

44 Z. X. Xue, S. T. Wang, L. Lin, L. Chen, M. J. Liu, L. Feng and L. Jiang, Adv. Mater., 2011, 23, 4270.

45 J. P. Zhang and S. Seeger, Adv. Funct. Mater., 2011, 21, 4699. 46 X. Y. Zhang, Z. Li, K. S. Liu and L. Jiang, Adv. Funct. Mater., 2011, 21, 4699.

47 M. Khosravi and S. Azizian, Microporous Mesoporous Mater., 2016, 230, 25. 